\title{
Conceptual Design of a 100 MW Electron Beam Accelerator Module for the National Hypersonic Wind Tunnel Program*
}

\author{
Larry X Schneider \\ Applied Accelerator Technologies Department, Sandia National Laboratories \\ Albuquerque, New Mexico 87185, USA*
}

\begin{abstract}
The National Hypersonic Wind Tunnel program requires an unprecedented electron beam source capable of 1-2. MeV at a total average beam power of 100-200 MW for several seconds. Direct-current electron accelerator technology can readily generate high average power beams, however, due to the nature of research and industrial applications there has never been a requirement for a single module exceeding approximately $500 \mathrm{~kW}$. Although a $100 \mathrm{MW}$ module is a two-order extrapolation from demonstrated average power levels, the scaling of accelerator components appears reasonable. This paper will present an evaluation of component and system issues involved in the design of a $100 \mathrm{MW}$ electron beam accelerator module with precision beam transport into a high pressure flowing air environment.
\end{abstract}

\section{INTRODUCTION}

The design of a Medium Scale Hypersonic Wind Tunnel (MSHWT) facility is being explored to address deficiencies in present ground test capabilities above Mach 8. Conventional wind tunnel techniques involving isentropic expansion from a high-pressure, high-temperature source can not support this hypersonic parameter space and operation time without introduction of substantial high-temperature material challenges. Radiative energy addition, a concept proposed by Princeton University [1], provides a potential means to extend wind tunnel technology above Mach 8 while preserving prototypic flight conditions in the test section. For an output section that allows $50-100 \mathrm{~cm}$ diameter test objects, approximately $100-200 \mathrm{MW}$ will need to be added to the airflow from an external power source. An electron beam guided into an air expansion nozzle is a potentially efficient means to add external energy to the flow [2]. Generating a continuous, multi-second, 1-2 $\mathrm{MeV}$ electron beam at this power level would represent a two order of magnitude increase in the power demonstrated by an electron beam accelerator in this class. The beam power of the MSHWT accelerator system would exceed the combined output power of the approximately 1000 industrial accelerators installed worldwide. An artist's concept of the MSHWT facility is shown in Fig. 1.

\section{ACCELERATOR SYSTEM DESIGN}

The MSHWT system is comprised of four subsystems: a high voltage DC power supply, electron injector and accelerating column, aerodynamic window, and a beam transport magnet system.

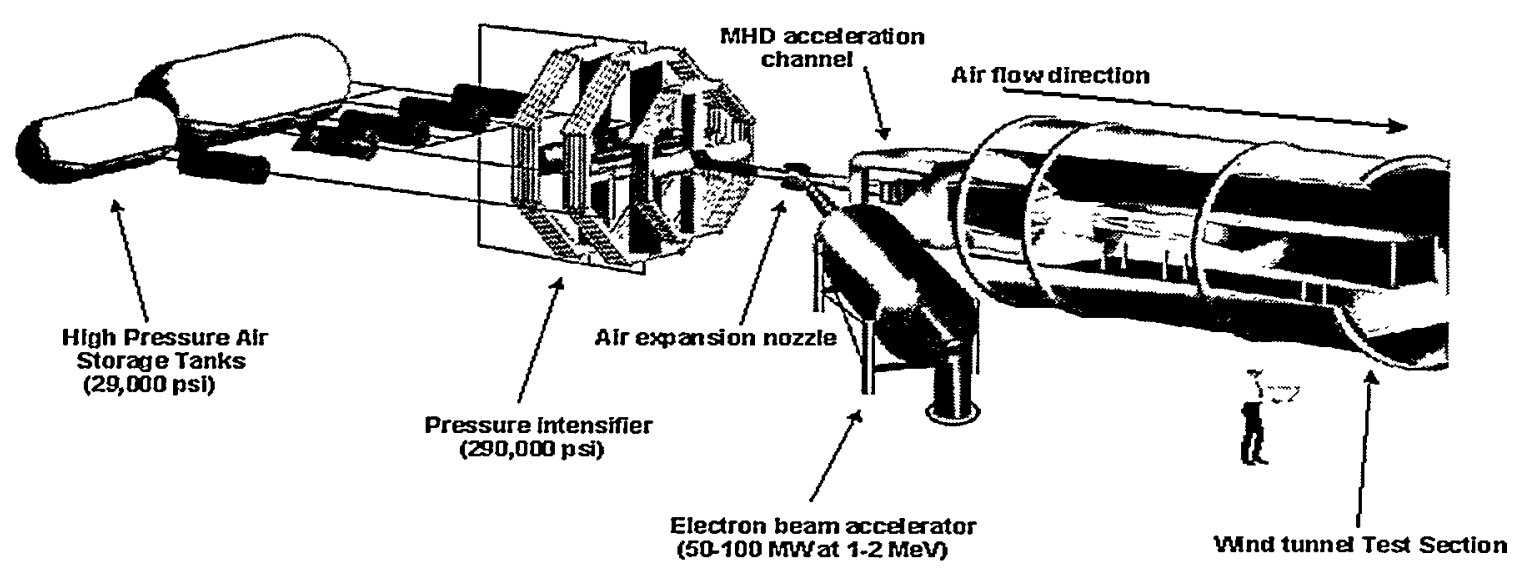

FIGURE 1. Artist's concept of a Medium Scale Hypersonic Wind Tunnel Facility. Electron beam accelerators play a key role for both energy addition into the nozzle and for enhancing the conductivity in a MagnetoHydroDynamic (MHD) afterburner.

* This work supported by the USAF and managed by the Amold Engineering and Development Center. 


\section{DISCLAIMER}

This report was prepared as an account of work sponsored by an agency of the United States Government. Neither the United States Government nor any agency thereof, nor any of their employees, make any warranty, express or implied, or assumes any legal liability or responsibility for the accuracy, completeness, or usefulness of any information, apparatus, product, or process disclosed, or represents that its use would not infringe privately owned rights. Reference herein to any specific commercial product, process, or service by trade name, trademark, manufacturer, or otherwise does not necessarily constitute or imply its endorsement, recommendation, or favoring by the United States Government or any agency thereof. The views and opinions of authors expressed herein do not necessarily state or reflect those of the United States Government or any agency thereof. 


\section{DISCLAIMER}

Portions of this document may be illegible in electronic image products. Images are produced from the best available original document. 
Scaling to the $100-200 \mathrm{MW}$ range will require a careful review of the physical limitations of each component from the birth of the electrons at the cathode through the acceleration phase and transport of the beam into the high-pressure expansion nozzle.

There are three major classes of electron beam accelerator systems that have the capability to scale to megawatt outputs at beam energies in the few $\mathrm{MeV}$ range. They are: Radio Frequency (RF), DirectCurrent (DC), and repetitive pulsed accelerator systems. Each technology base has intrinsic and practical limitations. The MSHWT application requires energy addition that is relatively constant with respect to the time scales involved in the dynamic air flow in the expansion nozzle. Conventional RF LINAC accelerator technologies can be designed to operate in a CW mode at a frequency from approximately $100-1000 \mathrm{MHz}$. Although this meets the temporal uniformity requirements, existing RF technologies are limited to an efficiency of approximately 50\%. Pulsed accelerator technologies, such as Sandia's RHEPP technology [3], has a relatively weak cost scaling 'relationship with output power. However, the efficiency of this and other repetitive pulse accelerators is presently limited to approximately $60 \%$. DC accelerator technology is attractive from several standpoints. Inductively coupled rectifying transformer systems offer simplicity of design, robustness, and a significant capability to scale cost effectively to very high power levels. For these reasons, inductively coupled rectifying transformer systems were selected for further analysis in the MSHWT facility conceptual design

Several DC accelerator power supply concepts were studied under a Sandia contract by the Delta Division of the Efremov Institute in St. Petersburg, Russia. The Delta group was selected due to their industrial experience with inductively coupled transformer accelerator systems. Fig. 2 shows a three-phase, ironcore, rectifying transformer concept for a $2 \mathrm{MeV}$, $100 \mathrm{MW}$ power supply. A $2 \mathrm{MeV}$ accelerator column and differential pumping system for a foil-less window is shown to the left of the high voltage power supply. Although the high voltage output must be insulated from the grounded core in this design concept, the coupling of flux from the primary windings around each vertical core to the high voltage secondary is very high. Closed core concepts can have electrical efficiencies $>95 \%$. The power supply and accelerator are insulated with SF6 and N2 and would use commercially available $25 \mathrm{kV}$ diodes in the rectifier assembly.

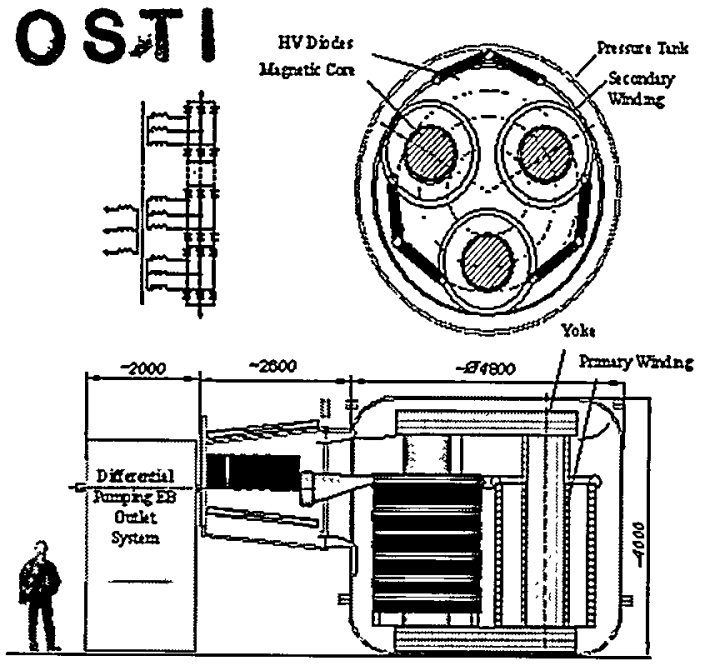

FIGURE 2. $2 \mathrm{MeV}, 100 \mathrm{MW}$ DC rectifying transformer power supply concept. Dimensions are in $\mathrm{cm}$.

\section{Injector Physics}

A $2 \mathrm{MeV}, 100 \mathrm{MW}$ accelerator module will require 50 amps of beam current. At an accelerating potential of $50 \mathrm{keV}$, this injector will generate a $2.5 \mathrm{MW}$ beam. A conventional Pierce-type injector can not deliver this current in a reasonable diameter beam due to space charge limitations. The high injector perveance (15.6 $\mu$ perv) will require a numerically designed geometry to optimize the space charge limited flow. Fig. 3 shows an injector design operating at $50 \mathrm{keV}$ and $50 \mathrm{~A}$ with a beam output diameter just under $3 \mathrm{~cm}$. The injector is immersed in a $100 \mathrm{G}$ solenoidal magnetic field in this simulation which was generated by a Poisson-solving code developed at Sandia. The electron source would be a standard thermionic cathode material such as Lanthanum hexaboride $\left(\mathrm{LaB}_{6}\right)$, which has ample current density and lifetime characteristics. The design shown in Fig. 3 operates at a conservative $2.5 \mathrm{~A} / \mathrm{cm}^{2}$.

After initial acceleration to $50 \mathrm{keV}$ in the injector, the next accelerating gap can also limit the total beam current due to space charge effects. Space charge limits in the first accelerating column gap can be estimated by modifying the planer Child-Langmuir equation to account for the injected $50 \mathrm{keV}$ beam. Enhanced flow is described by Eq. (1).

$$
\mathrm{j}_{\mathrm{enh}}=\mathrm{j}_{\mathrm{o}} \mathrm{F}(\chi) \mathrm{A} / \mathrm{cm}^{2}
$$

where $\mathrm{i}_{0}$ is the Child-Langmuir current, $\mathrm{F}(\chi)=\chi^{3 / 2}[(1-$ $\left.1 / \chi)^{3 / 4}+1\right]^{2}$ and $\chi=-\varphi_{2} /\left(\varphi_{1}-\varphi_{2}\right) . \quad \varphi_{1}$ is the beam energy entering the gap and $\varphi_{2}$ is the beam exit energy (injection energy + gap energy). 


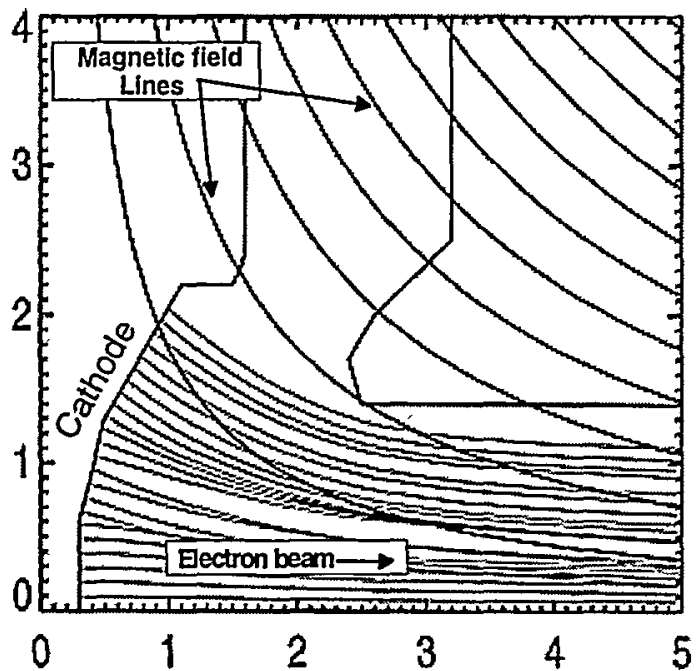

FIGURE 3. $50 \mathrm{keV}, 50 \mathrm{~A}$ injector. This cross-section is symmetric around the lower horizontal axis. Dimensions are in $\mathrm{cm}$.

Industrial accelerator columns routinely operate with electric field gradients of $1-1.5 \mathrm{MV} / \mathrm{m}$ on the ceramic insulators. For an accelerating gap in the column of $\mathrm{d}_{1}=5 \mathrm{~cm}$, a gap voltage of $\mathrm{V}_{1}=60 \mathrm{kV}(1.3 \mathrm{MV} / \mathrm{m})$, and a beam diameter of $3 \mathrm{~cm}$, the non-enhanced space charge limited current is only $9.7 \mathrm{~A}$. For a $50 \mathrm{keV}$ injected beam and a gap potential of $\mathrm{V}_{1}=60 \mathrm{kV}, \chi=$ 2.2 and $F(\chi)=8.5$. This allows a maximum current in the $3 \mathrm{~cm}$ diameter beam of $I_{o}=82 \mathrm{~A}$. The injected $50 \mathrm{keV}$ significantly enhances the flow in the first column gap. A similar gain is seen in the second gap $\mathrm{d}_{2}$. For a beam entering at $110 \mathrm{keV}, \chi=2.8$ and $\mathrm{F}(\chi)=14$. The maximum current space charge will allow in gap $d_{2}$ is $I_{2}=(9.7 \mathrm{~A})(14)=136 \mathrm{~A}$. As the beam accelerates past $500 \mathrm{keV}$ and becomes relativistic, the space charge limit raises significantly. Based on this space charge analysis, it is reasonable to envision a $100 \mathrm{MW}$ module at $2 \mathrm{MeV}$ or a $50 \mathrm{MW}$ module at $1 \mathrm{MeV}$.

In practice, there will be beam optics issues that impact the peak current capability of this accelerator column. At these power levels, beam losses in the column must be extremely low to prevent damage to the accelerating gaps and ceramic insulators. The beam divergence must be very low and beam halo that could impact the gap electrodes must be extremely well controlled. These issues could limit a system of this size to several amperes instead of 10's amperes. However, the MSHWT accelerator system will require that the cathode, injector, and accelerating column be immersed in an externally supplied axial magnetic field to mitigate magnetic mirroring effects. This axial solenoidal field will also serve to confine the beam as it leaves the finite divergence injector and accelerates through the column. The axial magnetic field is a key requirement to extend the beam current into the 10's of ampere regime.

\section{Beam transport to the nozzle}

This application requires that the electron beam travel from its origin in the vacuum insulated accelerator to a high-pressure expansion nozzle within the wind tunnel chamber. The beam will exit the high vacuum environment of the transport region through a aerodynamic window where it will then be guided and compressed to approximately $0.5 \mathrm{~cm}$ diameter in the Energy Injection Region (EIR) as shown in Fig. 4

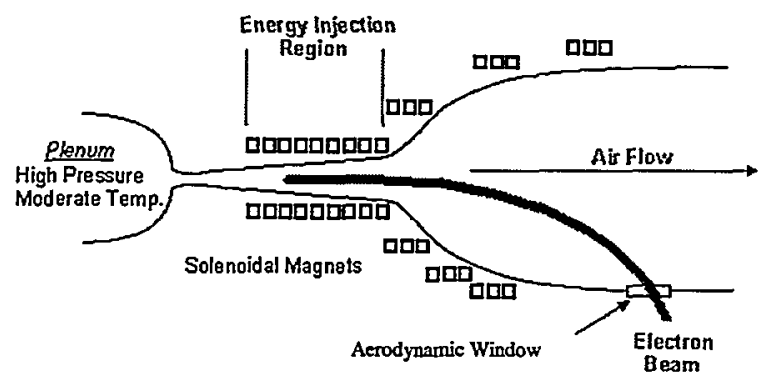

FIGURE 4. Cross-section of the expansion nozzle and energy injection region.

The region from the aerodynamic window to the entrance of the EIR will require modeling to evaluate the magnitude of gas breakdown in the approximate 1 atm environment that extends for about $0.3 \mathrm{~m}$. As the beam enters the $100 \mathrm{MPa}$ environment of the EIR, electron scattering, space charge, and charge migration issues will dominate the physics concerns in this region. At these high power levels, high energy electrons can not be allowed to impact the wall of the nozzle. The scattering beam must be compressed radially as it enters the nozzle using a series of multiTesla solenoidal magnets. Collisional effects in the high pressure nozzle have been modeled using Cyltran to set the magnitude of the solenoidal B-field in the EIR. Cyltran is a Monte-Carlo particle-in-cell code developed at Sandia that accounts for the collision of electrons with gas atoms in this region. A 20 Tesla magnetic field was required to minimize beam loss to the nozzle walls. Further modeling in the EIR will be required as Cyltran does not account for beam selffields or the effects of electron induced gas chemistry.

Once the peak B-field strength is set in this region of the MSHWT system, the B-field profile back to the cathode can be designed. The $20 \mathrm{~T}$ magnetic field is sufficiently high to reflect back all the $1-2 \mathrm{MeV}$ electrons entering this field if those electrons are born in a zero-field region at the injector. This mirroring 
effect can be estimated by considering the electron's gyrofrequency and conservation of energy as the particle converts azimuthal velocity to axial velocity. The maximum solenoidal B-field that an electron can propagate into (starting from a zero field) can be estimated as,

$$
B_{\max }=\underline{2 c_{c}} \underline{\underline{m}_{e}} \underline{q_{e}} r \underline{\beta}
$$

where $B_{\max }=B$-field strength ( $T$ ) and $r=$ beam radius (m). For a $2 \mathrm{MeV}$ electron beam starting with a $0.5 \mathrm{~cm}$ diameter outside the solenoid, mirroring will occur at a field strength above approximately $6.6 \mathrm{~T}$. To overcome this effect the electrons must be immersed in a continuous solenoidal B-field back to cathode electron source. Determining the magnitude and optimum profile of the axial magnetic field back to the cathode will require numerical modeling techniques. The minimum field strength needed at the cathode can be estimated as,

$$
\mathrm{B}_{\mathrm{o}} / \mathrm{B}_{\mathrm{m}}=\sin ^{2} \theta
$$

where $B_{o}=$ minimum field strength, $B_{m}=$ maximum field strength, and $\theta=$ divergence angle. The high perveance injector configuration and imperfect electric fields through the accelerator column will produce a beam with 10's milli-radian divergence at the exit of the accelerator column. If we assume $40 \mathrm{mrad}$ and use $B_{\max }=20 \mathrm{~T}, B_{0}$ will need to be on the order of $0.03 \mathrm{~T}$ at the cathode and increase to $20 \mathrm{~T}$ at the EIR. Minimizing the divergence in the injector and accelerator column will be important to reducing the cost of the integrated magnet system.

\section{Aerodynamic window}

Conventional foil windows will not survive the heating from a multi-second beam much above a few $\mathrm{mA} / \mathrm{cm}^{2}$. A foil-less aerodynamic window will be required to transition the beam from the vacuum line into the approximately 1 atm environment just downstream of the expansion nozzle. An exit aperture of $<1 \mathrm{~cm}$ diameter will be required to inject beam into the wind tunnel chamber. This can be accomplished through conventional differential pumping techniques or potentially through other techniques that can reduce the overall high volume vacuum pumping requirements and system cost. A plasma porthole [4], a high temperature, low density, high viscosity plasma channel, is being evaluated for the high pressure stage. A pressure reduction from approximately 1 atm to approximately $350 \mathrm{mT}$ Torr can occur in this stage, leading to a reduction by a factor of $>200$ over standard differential pumping techniques.

\section{SUMMARY}

Demonstrated industrial DC accelerator technology forms the basis of the MSHWT accelerator concept described in this report. Scaling issues in extrapolating this technology base have been examined at a conceptual level. This work has not identified any fundamental physics that would prevent a system of this magnitude from being developed and fielded into a reliable hypersonic ground test facility. However, several areas of this system require further analysis. The next phase of this system design will include analysis and simulations in key risk areas such as the injector, accelerator column, and beam transport in the non-vacuum environment. Many of these issues will be explored in the next several months.

Proof-of-principal energy addition experiments, in conjunction with Princeton University, have already been completed at Sandia at a power level of $150 \mathrm{~kW}$ into a flow gas nozzle [4]. The project team is currently preparing for a $1 \mathrm{MW}$ energy addition demonstration in the summer of 2001.

\section{ACKNOWLEDGEMENTS}

The National Hypersonic Wind Tunnel team consists of members from Princeton University, MSE Technology Applications, Inc., the USAF Arnold Engineering and Development Center (AEDC), Lawrence Livermore National Laboratories, and Sandia National Laboratories. The artists concept shown in Fig. 1 was generated by AEDC. The required beam conditions and magnetic field requirements were generated by Dr. Ron Lipinski of SNL based on thermodynamic conditions generated by Princeton University. The injector simulation shown in Fig. 3 was generated by Barry Marder, SNL.

\section{REFERENCES}

1. R. B. Miles, "Radiatively Driven Hypersonic Wind Tunnel", AIAA Joumal, Vol. 33, No. 8, August 1995.

2. R. J. Lipinski, "Conceptual Design for an Electron-Beam Heated Hypersonic Wind Tunnel," Sandia National Laboratories report, SAND97-1595, July 1997.

3. L. X Schneider, "Repetitive high energy pulsed power technology development for industrial applications", Application of Accelerators in Research and Industry, AIP, CP392, 1997.

4. A. Hershcovitch, "High-pressure arcs as vacuumatmosphere interface... ", J. Appl. Phys. 78 (9), Nov. 1, 1995.

5. P. Barker, “A $150 \mathrm{~kW}$ Electron Beam Heated Radiatively Driven Wind Tunnel Experiment", presented at the AIAA Conference, Jan. 2000, Reno, NV.

Sandia is a multiprogram laboratory operated by Sandia Corporation, a Lockheed Martin Company, for the United States Department of Energy under contract DE-AC04-94AL85000. 